\title{
Design of Disaster Relief Robot Assistant
}

\author{
Chao Liu, Guang-xian Xu \\ School of Electronic and Information Engineering \\ Liaoning Technical University \\ Huludao, China \\ liuchao@Intu.edu.cn
}

\author{
Feng Gao, Nan Xu \\ School of Electronic and Information Engineering \\ Liaoning Technical University \\ Huludao, China \\ liuchao6655@gmail.com
}

\begin{abstract}
In the world, the disasters often occur due to natural disasters, terrorist activity and other reasons, and the rescuers only have a very short time in the ruins to find survivors in disaster relief, or their survived chances are nearly nil. In this dangerous environment, robotics can help rescued workers. Autonomous intelligent robots will be used for search and rescue. It responds to a lot of work effectively, quickly and reliably. Robot will be mastered by the MCU of S3C2410. Specific function through the UDP controls the robot via a wireless network card to receive the control command, and the camera captures the real-time video, life detector, temperature and humidity and GPS coordinates transferred back to host system to achieve the purposes of relief disaster.
\end{abstract} WiFi

Keywords-GPS; UDP; QTE; Disaster Relief Robot Assistant;

\section{INTRODUCTION}

In recent years, with the rapid development of microelectronics technology, computer technology, software technology, sensor technology and automatic control technology, the information flow becomes as the main characteristic of mechatronics. Its products realize the automation, the digital and the intelligent. At the same time, a qualitative leap is achieved on the performance and the function. Robot is a typical mechatronic product, all countries pay attention to the development of the robot when the robot technology is associated with the relief disaster, the robot technology for the relief disaster. Its objectives are earthquakes, mine disasters, fire, flood and other areas, but the application of the robot technology is not mature in the earthquake relief. The search for disaster occasions such as the earthquake ruins and the disaster relief robot assistant will be achieved in the system, which is divided into two parts: the control display side and the walking exploration robot.

The research of the system applies the complex way of the wheeled and the legged, that is the autonomous mobile robot with the bionic wheeled and legged with six-foot, which is used to imitate the leg structure of the ant and its walking posture, and it is a special robot for relief disaster. The robot's movement is achieved by the coherent action of the legs, the design of six-legged strengthens the stability of the robot's walking, and that design adapts to the unstructured environment, so that it's possible to walk on the road in the bumpy conditions, and a rapid wheeled movement is used on the smooth surface, in a word, it is better than the wheeled and the tracked.
The display side of the system control sends the control command to the detecting robot by using the WiFi wireless network, and then the detecting robot receives the command, so that the walking in and on the ruinous surface can be obtained. The detecting robot sends the environmental conditions including the video and the temperature probed by the camera and the GPS coordinates to control display side by the wireless network card. Through the screen display, we can found that the wounded and their position in order to guide rescue workers.

\section{SYSTEM SCHEME}

\section{A. Overall Layout}

The system including two parts that are control display terminal and walk detection robot. The control display terminal send control command, transmitting walk detection robot by using WiFi Wireless LAN to realize real-time control, then walk detection terminal by using camera, temperature and humidity, methane sensor, GPS and so on. The information which collected by those use Wireless LAN to send back to the control display terminal, to realize realtime environment detection. Figure 1 shows the whole structure.

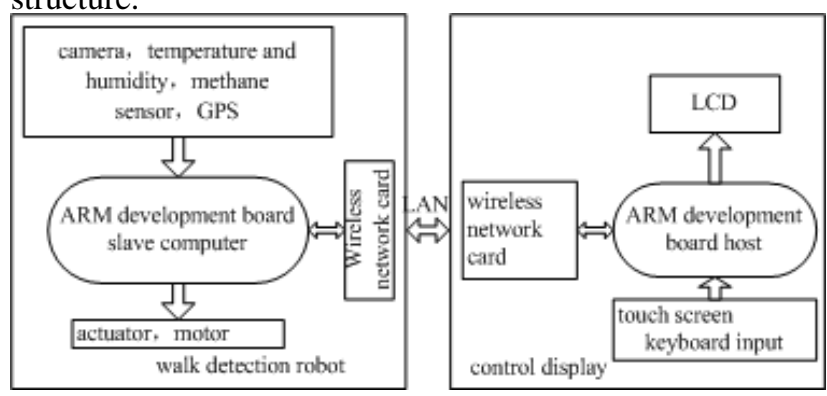

Figure 1. general design structure

The robot designed in this paper use composite mode that is wheel merge leg. That is round leg autonomous mobile robot.

The analysis of the basic structure of six legs rescue robots as bellow:

- $\quad$ Body used to fix and install other parts.

- Travel agencies mainly composed by dc motor, driving wheel and reducing gear train. In order to increase driving capability, decreasing demands of motor performance, cancel idle gear. Robots control speed of driving motor simultaneously, make 
steering motor angle control, which, driving wheel by worm and worm gear use six dc motors to decelerate driving, ten motors control six wheel-leg respectively, using independent way to realize steering motion, Velocity measurement make realtime feedback regulation.

- $\quad$ The cross obstacle mechanism of main shake leg and side shake leg mainly completed by rocker arm which cans rotary 180 degree in vertical plane. When meet disorder or special condition, by control rotation angel of shake leg can make body at different state, to fit running framework obstacle negotiation, so to increase crossing ability of the robots.

\section{B. System Function}

This system use semi-automatic working mode, combined with real-time control and real-time data acquisition aided rescue robot, it can into regional or dangerous area which first-aid staff can not into, it can feedback real situation to master port of safety zone(control display terminal), on flatland, its walk with four wheels, the speed is fast. On unsmooth waste area, it can throw over different terrain by four legs or six legs. At the barrier like step, it also can throw over the obstacle, this can improve success rate of rescue, improve efficiency.

\section{C. hardware and software platform}

The hardware platform of control display terminal use Intel PXA270 processor. Using WiFi wireless network card to transfer all data, guarantee wireless LAN stable and high efficiency. Wireless transmission rates $54 \mathrm{Mbps}$ meet video and data transmission.

The hardware platform of walk detection robot use S3C2410 processor, its working frequency is $203 \mathrm{MHz}$. The hardware system is composition of temperature and humidity acquisition, light intensity acquisition, rudder controller.

The part of temperature and humidity acquisition use SHTxx chip digital temperature and humidity acquisition sensor, this sensor use 2-wire communication mode and its low power, fast reaction. The measuring range of temperature is 55 degrees below zero to 125 degrees; measured temperature resolution is up to 0.0625 degree. Measurement of humidity is $\pm 5 \% \mathrm{RH}$.

Light strength acquisition depends on photo resistor collect strong and weak light, after two grade amplification of two triode, transmit analog to AD converter of S3C2410, after transfer analog to digital quantity judge the size of light intensity.

Self-made rudder controller use ATmega16. Using it to output 11 channel PWM control signal, 6 channel motor control signal and 4 line stepping motor control signal, in which 6 channel motor control signal use L293D as motor driver IC. By output different signals can control each motor's steering and stop, so realize various motion of robot. Rudder controller use serial port and S3C2410 to communicate.

Software platform use Linux operating system, QTE make upper software and interface. Linux operating system kernel version is Linux 2.6.24, QTE use QtEmbedded-4.5.3. Cross-compiling environment use arm-linux-gcc-3.4.6-glibc2.3.6.

LCD is 3.5 inch $320 \times 240$ TFT, touch screen FM7843 driving. USB camera use VIMICRO 301, video transmission rate is 20 frames/s. Video resolution is $320 \times 240$.

\section{GAIT CONTROL}

Six-foot rescue robot is composed of body and six legs. The robot machine is a regular platform; each leg is connected with the body through the hip joints. The walking legs of robot are composed of a planar linkage, hip joints and casters.

\section{A. Gait control implementation of six-foot rescue robot}

Based on generation gait algorithm, we can control the six-foot rescue robot generate gait automatically on the uneven ground, including the leg movement posture, stride and swing leg trajectory.

In order to simplify the control, on the basis of considering the robot ability to adapt to the uneven ground situation, set a particular value of the ground's non-flatness $\Delta v$, and set the maximum height $\Delta y$ on the condition of wheel robot walking, set the maximum height of the robot obstacle avoidance $\Delta x$, when $\Delta v<\Delta y$, the robot moving straight or turning by using the wheel; when $\Delta y<\Delta v<\Delta x$, the robot use the pattern of swinging leg climb the obstacle; when $\Delta v>\Delta x$, the robot use the pattern of swinging leg avoid the obstacle.Figure.2 shows Gait control of robot obstacle crossing. 


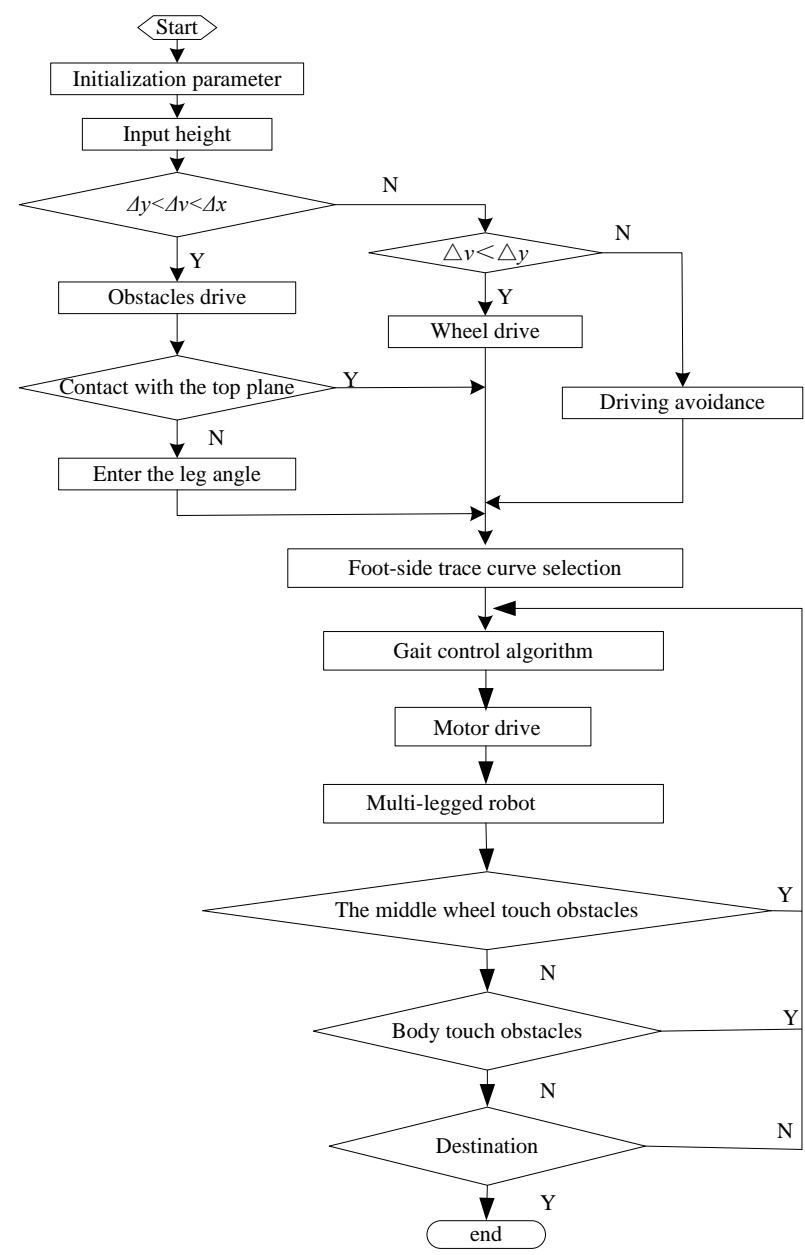

Figure 2. Gait control of robot obstacle crossing

B. the leg movement analysis of six-foot robot obstacle crossing

The course gait of obstacle crossing is the robot leg around the body in the vertical plane, as shown in Figure. 3. Leg swing angle measured according to the height of obstacles, robot drive forward when swinging leg contact the top of obstacles, and drive forward the next set of legs, if the body touch obstacles, the driving wheel reverse so that the body left the obstacles, the next set of swinging leg go on lifting, control process is same with the first set of legs execution process.

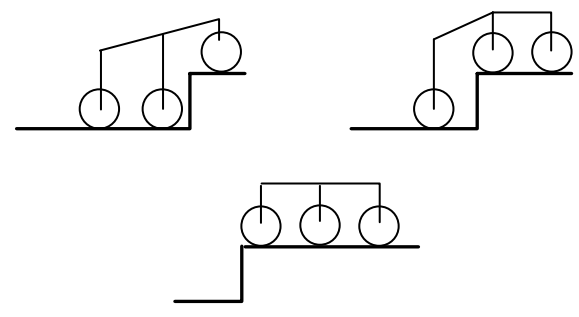

Figure 3. the course of six-foot robot crossing obstacles

\section{DESIGN OF SOFTWARE SySTEM}

The design of software system is divided into three parts: The software design of Control display terminal, the Software design of walking exploration robot and Servo Motor Controller.

\section{A. the software design of Control display terminal}

Graphical interface of control receiver is made by QT. It is used to display the received video, Temperature and humidity, GPS information and other Environmental parameters. The main design of the interface is shown in figure 4.

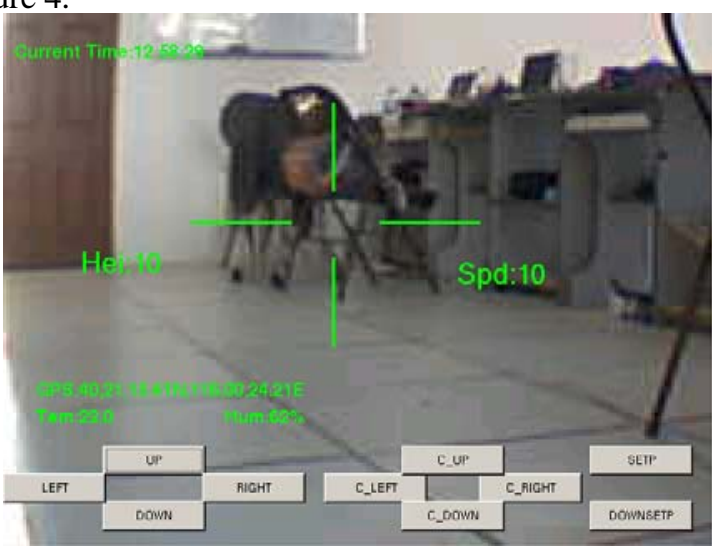

Figure 4. Main interface design drawing

The interface shows us the system time, height of the location of walking exploration robot, walking speed, GPS information, temperature, humidity and other information. It provides ten buttons below the interface. They are used to control the motion of the robot, rotation of camera, get over the barriers and other functions. The system uses touchbutton and keyboard redundancy design, which ensures the reliability of the system.

The video is conveyed back to control display terminal through the UDP protocol of Wireless Network. The key codes of UDP video transmission Sender are as follows:

socketC = socket(AF_INET, SOCK_DGRAM, IPPROTO_UDP);

serverAddr.sin_family = AF_INET;

serverAddr.sin_port = htons(5679);

serverAddr.sin_addr.s_addr = inet_addr("10.10.86.21");

sendto(socketC,sendbuf,sizeof(sendbuf),0,(SOCKADDR

*)\&serverAddr,sizeof(serverAddr));

closesocket(socketC);

send buffer is the address of one frame of JPG video streaming which acquired by V4L (Video4Linux).

The key codes of UDP video transmission receiver are as follows:

socketC = socket(AF_INET, SOCK_DGRAM, IPPROTO_UDP);

serverAddr.sin_family = AF_INET;

serverAddr.sin_port = htons(5679);

serverAddr.sin_addr.s_addr = htonl(INADDR_ANY);

bind(socketC,

(SOCKADDR*)\&serverAddr, 

$0,0)$;

int nRet = recvfrom(socketC, recvbuf, sizeof(recvbuf), 0 ,

The receiver is different from the transmitter. Port number must be bound at the receiver. And then it begins to receive data. The received data is showed by Label of QT. The transmission of video is smooth and without delay in the actual experiment. So we can ensure that the operators can clearly see the real-time video of the area to be detected.

\section{B. Software Design of the Walking robot}

The process of video programming is as follows:

- Turn on the video device by OPEN function which is provided by drivers.

- Read the device information by Read function which is provided by the drivers

- Change the current settings of device (The operation can be omitted) by octal function which is provided by the drivers. Such as the operation of resolution of the collected images.

- Capture video. The two methods of capture video are: a. memory mapping .b. Read directly from the device.

- Processing the collected video. This is good for next step. Such as compress data and so on.

- Turn off the video equipment.

\section{Programming of servo controller}

The programming of servo controller is divided into two parts: serial communication section and PWM output section.

Servo controller uses ATmega16 as its control chip. The main function of ATmega16 is to control robots by PWM output and receive the instructions from ARM. The AVR data is received by using interrupt mode. Key codes are as follows:

\section{SIGNAL(SIG_UART_RECV )}

\{

static unsigned char $\mathrm{i}=0$, $\arg 0$, $\arg 1$;

char temp;

while ( !(UCSRA \& $(1<<$ RXC)) ); // Waiting for receiving data

$$
\begin{aligned}
& \text { temp= UDR; } \\
& \text { if(temp='D'||temp='J'||temp='B' }\{ \\
& \mathrm{i}=1 \text {; } \\
& \arg 0=\text { temp; } \\
& \text { \}else if(i }=1)\{ \\
& \text { arg1=temp; } \\
& \mathrm{i}=2 \text {; } \\
& \text { \}else if(i }=2)\{ \\
& \mathrm{i}=0 \text {; } \\
& \text { if }\left(\arg 0={ }^{\prime} D^{\prime}\right)\{ \\
& \text { change(arg1,(unsigned char)temp); } \\
& \text { \}else } \\
& \text { if }\left(\arg 0={ }^{\prime} B '\right)\{ \\
& \text { bjdj_run(arg1,(unsigned char)temp); } \\
& \text { \}else } \\
& \text { if }(\arg 0=\text { 'J') }\{ \\
& \text { gocontrol(arg1,(unsigned char)temp); } \\
& \text { \} }
\end{aligned}
$$

\}

Data transmission between the host computer P270 and servo controller is divided into three kinds. One is the servo control, the second is a stepper motor control, and the third is geared motors. The agreement of the lower computer and the host computer is that each sent data is beginning with $\mathrm{D}, \mathrm{B}$, and $\mathrm{J}$.

The output of 11 channels PWM depends on completion of the timer interrupt .Key codes are as follows:

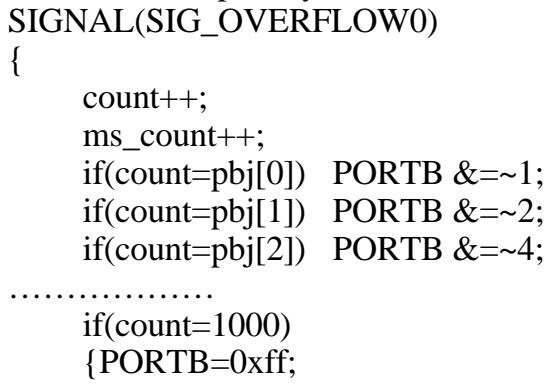

Analog output of PWM with a timer is a common method in microcomputer. It will complete PWN servo control signal of $50 \mathrm{~Hz}$ and a PWM cycle when performs a thousand times interrupt.

\section{CONCLUSIONS}

The paper is mainly introduced the walking agency of the disaster relief robot with six-foot and its work environment are introduced respect, and the gait analysis of the disaster relief robot with six-foot is gotten in this paper, so that the obstacle avoidance of the six-foot disaster relief robot can be controlled. The detecting robot is shown in figure 5.

Due to the system complexity of $t$ the six-foot disaster relief robot and the research is limited by the time and the author's ability, the study in the paper is preliminary, many aspects have not been fully taken into account, the deficiency are as follows:

- This study assumes that the robot transmits the data by $\mathrm{WiFi}$, and provided that there is no interference for the wireless signal in the transmission environment.

- There is no analysis of the kinematics model for the robot, and the control theory can not accurately be tested in the work environment.

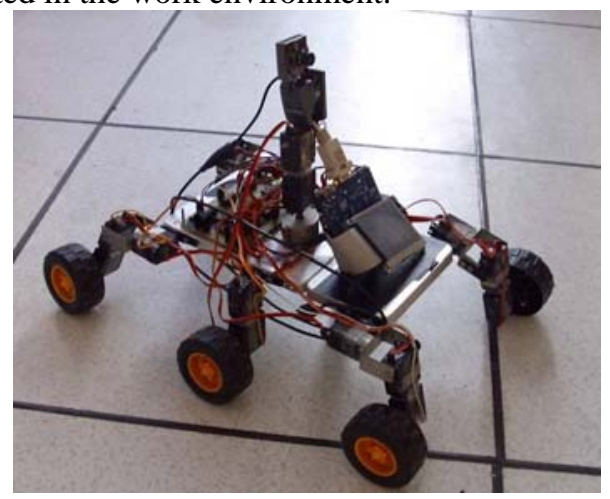

Figure 5. the physical map of the detecting robot 
Based on the practical test of the system, video transmission is smooth, the instruction of sending and receiving is normal, the obstacle and the obstacle avoidance action can be completed. The acquisition parameters can be finished according to the instructions, and a method is proposed for the design of disaster relief robot. Meanwhile, the author will study the WiFi data relay module, the image transmission with higher-resolution and the design of multichannel redundancy, multiple power structure, so that the disaster relief robot can be used farther in more work environment.

\section{REFERENCES}

[1] Luo Zhizeng, Jiang Jingping. Robot feeling and information fusion. Beijing: Mechanical Industry Press. 2002

[2] Cai Guoxing. Principle and Application of the robot. Central South University Press. 1988

[3] Chen Xuedong, Sun Yu, Jia Wenchuan. Multi-legged walking robot motion planning and control. Huazhong University Press. 2006.6

Article in a journal:
[4] Wan Hong. The obstacle analysis and research of uneven ground sixlegged autonomous mobile robot. Nanjing University of Science. 2006.5

[5] Alexander R M.1993.Gaits of Mammals and Trestles. IEEE J. of the Robotics Society of Japan, 11(3): 314-319

[6] Brooks R. 1986. A Robust Layered Control System for a Mobile Robot. IEEE J. of Robotics and Automation. 2(1): 14-23

[7] Wang Jinsong, Rong Songnian, Zhang Bopeng, Comprehensive double three-legged walking robot (I)--Walk theory, organization and control system. Tsinghua University: Science and Technology. 1994. (2): $102-107$

[8] Liu Haibo, $\mathrm{Gu}$ Guochang, Zhang Guoyin. Intelligentrobot architecture classification. Harbin Engineering University. 2003. 24(6): 664-668

[9] Jia Wenchuan. Multi-legged walking robot bionic control. Huazhong University of Science. 2006.4

Article in a conference proceedings:

[10] Akimoto K, Watanabe S, Yano M. 1998. An Insect Robot Controlled by Emergence of Gait Patterns. AROB III'98 Oita, Japan: 110-113

[11] Bai Shaoping, Low H, Seet G, et al. 1999. A New Free Gait Generation for Quadrupends Based on Primary/Secondary Gait. Proc. of the 1999 IEEE Int. Conf. on Robotics and Automation. Detroit, Michigan: 1371-1376 